\title{
LES PROPRIÉTÉS TEMPORELLES DES NOMS ABSTRAITS À L'EXEMPLE DES FORMATIONS À PREFIXE -INTER
}

\begin{abstract}
Nkollo Mikołaj, Les propriétés temporelles des noms abstraits à / exemple des formations à prefixe "-inter " [Temporal features of French abstrac1 nouns. The case of inter-prefix forms]. Studia Romanica Posnaniensia, Adam Mickiewicz University Press. Poznań, vol. XXXII: 2005, pp. 65-75. ISBN 83-232-1465-4. ISSN 0137-2475.

The present paper deals with temporal properties of noun phrases whose head is a nominal lexical item with an inter-affix. The whole description is based on the linear concept of time. The key notions used in the following account are precedence in time, momentary thing, interval and coincidence. They originate from mereology. If the inter-prefix is added to a continuous nominal root, the whole noun phrase designates two identical states of affairs or two homogenous activities. The temporal relations holding between them are characterized in terms of overlapping of two intervals. As for nouns that represent non-continuous notions, the inter-affixation results in naming two simultaneous events. The chronology of the latter is described in terms of coincidence of two momentary things. The paper comprises necessary definitions and numerous examples.
\end{abstract}

Les études sémantiques consacrées à l'affixation se concentrent généralement sur les modifications lexicales qu'entraine l'adjonction d'un morphème à une base dérivationnelle. Les changements consécutifs à l'introduction d'un nouvel élément dans la structure de l'expression de départ sont, la plupart du temps, caractérisés en termes de l'enrichissement lexical de celle-ci. En outre, les linguistes s'intéressent aux mutations qui se produisent au niveau syntagmatique à la suite de l'agglutination d'un affixe à la base dérivationnelle (Jackendoff, 1975: 649-652). Cette direction de recherches est certainement justifiée, mais ses résultats ont le défaut d'être incomplets et d'occulter certains aspects du problème. D'autres types de changements imputables à l'adjonction d'un affixe à des unités existant déjà dans le lexique méritent, eux aussi, qu'on leur prête attention.

Les réflexions qui suivent se rapportent à un groupe peu nombreux de substantifs. Cette indigence quantitative va pourtant de pair avec l'homogénéité séman- 
tique des unités lexicales appartenant à la classe en question'. La présence dans leur structure du formant inter- est révélatrice du rôle qu'assument dans la langue les morphèmes affixaux. Loin de se borner à ajouter un élément lexical additionnel au signifié de l'expression de départ, ils influencent les propriétés syntaxiques et les caractéristiques temporelles de la notion représentée par la base dérivationnelle. Ainsi l'article qui suit s'oppose-t-il aux procédures mises à contribution dans les manuels d'enseignement universitaire où il est courant, lorsqu'on parle de dérivation, de se borner à une présentation énumérative des affixes et d'accompagner ceux-ci de leurs paraphrases (cf. les reformulations du type : pré- «l'antériorité », re- "de nouveau, une nouvelle fois », etc.). Le mieux qu'on puisse espérer, après avoir appliqué ce type de méthodes, est une caractérisation sémantique vague et toujours approximative du sens du terme modifié.

Puisque les traits sémantiques de toutes les formations nominales à préfixe inter- ne s'équivalent pas, il convient de choisir celles qui seront soumises à l'analyse dans cet article. La délimitation des candidats au corpus s'appuie en premier lieu sur la segmentation des unités, donc sur une procédure par excellence traditionnelle. L'examen de la structure morphémique des expressions incorporant inter- est révélateur des problèmes relatifs à la motivation de cette classe de signes. Les dérivés avec le préfixe en question mettent le chercheur devant une alternative. Soit la signification de l'expression amalgame les propriétés sémantiques du préfixe et celles d'une série de bases dérivationnelles existant déjà dans le système lexical français, soit le sens du signe ainsi formé n'a rien à voir avec cclui qu'apporte chacun de ses éléments constitutifs.

Avant de passer au problème de la motivation sémantique des dérivés incorporant inter-, il convient de s'interroger sur leurs caractéristiques structurales. L'observation des unités lexicales où l'on reconnaît la présence de ce morphème montre qu'il s'adjoint très souvent à des noms fonctionnant d'une façon autonome dans le lexique français. C'est ainsi qu'on retrouve dans cette langue les expressions comme interdépendance $(<$ dépendance < dépendre), interchangeabilité $(<$ changeabilité < changer), interpénétration $(<$ pénétration $<$ pénétrer), interconnexion $(<$ connexion < connecter), etc. Parfois, le mécanisme qui sous-tend la création de ces noms est plus compliqué, comme ceci a lieu dans intervention ( $<$ intervenir), interruption $(<$ interrompre), interrogatoire $(<$ interroger $)$, interdic-

'L'apparicment cntre le nombre d'unités lexicales et l'homogénéité (ou son manquc) de leurs propriétés sćmantiques n'a pas de quoi surprendre, car une des lois de la statistique linguistique édicte quc plus le nombre d'éléments appartenant à une classe est élevé, plus la diversification des formes à l'intérieur de celle-ci est grande et moins les sens que l'on y reconnaît sont réguliers. Le bien-fondé de ce principe est confirmé, entre autres, par la diversité des effets sémantiques observables dans la classe de verbes à préfixe $r e$ - (ct ses allomorphes). Bien qu'on s'accorde à voir dans le sens des verbes dont cet élément fait partie I'information sur la modification, le changement, etc.. nombreux sont les cas et les emplois où la valeur de ce formant est peu manifeste (rassembler, renier, regarder, ravaler, raccourcir, efc.). En outre, la valcur emblématique de répétition, imputable à la présence de re-, connaît unc certainc gradation (cf. la liste de Jalenques, $2002: 78$ ). 
tion (< interdire), etc. Ces exemples montrent qu'il est la plupart du temps possible de reconstituer, directement ou non, la forme de la base dérivationnelle. Seuls quelques termes s'écartent de cette régularité (cf. interview, interception, interstice). La motivation formelle est donc très manifeste.

Il en est autrement de la motivation au niveau sémantique. En effet, la signification de la grande majorité des noms ainsi formés, après l'adjonction de inter-, n'est pas modifiée d'une façon prévisible. Ceci veut dire que le sens du signe issu de la combinaison de deux éléments n'est pas la somme des sémantismes du préfixe et du nom auquel celui-ci a été ajouté. Si l'on s'accorde à attibuer à inter- les sens " au milieu de » et "réciprocité ", on voit que l'une et l'autre valeur font défaut à des unités comme interdiction, interjection, interprétation, intercession, etc. Or, il y a disparité entre la dérivation formelle et la dérivation sémantique. Pourtant, certains noms incorporant le morphème en question sont sémantiquement réguliers. C'est ainsi que le sens «au milieu de» (spatial et temporel) s'observe dans interposition, intervention, interlude (à côté de prélude), intertitre, intermède, etc. Le formant inter-ajoute à la base dérivationnelle la valeur de réciprocité dans interchangeabilité, interdépendance, interaction, intersection, interpénétration, intercommunion, interversion (dans certains emplois seulement), interconnexion.

Bien que les noms appartenant à l'une et à l'autre classe soient sémantiquement et formellement motivés, leurs propriétés syntagmatiques ne se recouvrent pas. Là où le formant ajoute à la base dérivationnelle un sens spatial ou temporel, il existe plusieurs façons de saturer les positions d'arguments ouvertes par le terme déterminé, cf. L'intervention de l'ONU dans le conflit rwandais, L'intervention de l'ONU entre les deux fractions, L'intervention de l'ONU dans le conflit qui oppose le gouvernement à la guérilla maoïste. Ces syntagmes permettent de s'apercevoir de la multiplicité des variantes diathétiques réalisables auprès des noms prédicatifs appartenant à cette classe. Les caractéristiques syntagmatiques des unités lexicales auxquelles inter-confère un sens réciproque sont différentes. Les noms ainsi modifiés sont toujours dyadiques, ce qui veut dire qu'ils postulent la présence obligatoire de deux arguments appartenant à un seul ensemble (donc ontologiquement homogènes). Par conséquent, leur combinatoire syntaxique est plus rigide. En termes de la théorie des relations, les signes incorporant le préfixe inter- à valeur réciproque désignent des relations symétriques (cf. Marek et Onyszkiewicz, 2001 : 37-38). La définition formelle de celles-ci est présentée ci-dessous.

$$
\text { 1. } \operatorname{sym}(X)=\mathrm{df} \forall x \in X \forall y \in X\langle x, y\rangle \in R \rightarrow\left\langle y, x>\in R^{2}\right.
$$

${ }^{2}$ La formule $\langle x, y\rangle$ ne doit pas être confondue avec la notation standard (réalisée à l'aide des accolades) d'un ensemble $\{x, y\}$ parce que les statuts ontologiques de l'une el de l'autre different sensiblement entre eux. Tandis que l'axiome d'extension permet d'identifier l'ensemble $\{x, y\}$ à $\{y, x\}$ ou à $\{x, x, y, y, x\}$, il n'en est pas de même de l'entité représentéc par $\langle x, y\rangle$. Celle-ci est un couple ordonné. Ses éléments ne sont pas permutables. Il n'est pas non plus possible de les répéter. Le recours aux ensembles ordonnés de $n$ ćléments est nécessaire lorsqu'on veut définir ce que c'est qu'une 
Pour que cette formule puisse être appliquée à l'analyse des syntagmes nominaux d'une langue naturelle, il faut procéder au déchiffrement des signes qui la constituent. Les lettres $x$ et $y$ représentent les variables individuelles. Autrement dit, ce sont les symboles capables de désigner, chacun, un des éléments d'un ensemble. Il est nécessaire de souligner qu'une variable individuelle ne réfère pas à tous les membres de cet ensemble, mais à un objet non spécifié qui en fait partie (cf. Bańczerowski, 1980:12 "Variables are [representatives of] names denoting any one of an entire set of objects. This does not mean that a variable refers to all the elements of a set at once, but to a single but unspecified element of a set. Thus, variables denote arbitrary objects of a previously determined domain, called the domain of variability"). Il existe deux façons d'attribuer à une variable sa dénotation. La première solution consiste à lui substituer une constante, c.à.d. à spécifier expressément ce qui est représenté par la variable (p. ex. Max pour $x$ et Jacqueline pour $y$ ). L'autre façon de préciser sa valeur référentielle est due à l'emploi des opérateurs. Ceux-ci servent à lier les variables présentes dans la fonction propositionnelle soumise à la transformation. Ces deux opérations permettent de transformer la fonction (séquence de symboles contenant les variables) en une proposition au sens logique du terme (suite de signes, douée d'une valeur de vérité, c.à.d. vraie ou fausse). Par la suite, la transformation réalisée à l'aide d'une de ces opérations sera nommée saturation. $R$, variable prédicative, est un symbole qu'il est possible de saturer au moyen d'une expression (nom, adjectif, verbe, collocation verbo-nominale, etc.) précisant la nature du rapport existant entre $x$ et $y$ (ou entre les référents des noms qui ont été introduits à leur place).

La réciprocité qui caractérise les noms français à préfixe inter-est décisive du point de vue de la description des rapports chronologiques signifiés par les syntagmes dont ces termes sont les déterminés absolus. Puisqu'on parle, lorsqu'on a affaire aux expressions nominales incorporant ce formant, d'actions et d'états de choses rapportés à au moins deux individus distincts (ce qui est dû à la nature des relations symétriques qui n'acceptent pas qu'il y ait seulement un actant), il convient de s'interroger sur ce qu'il en est des rapports temporels entre ces événements. En cffet, il découle de L'intersection de cette route et de l'ancienne rue Guilbert que l'entrecroisement n'a pas lieu uniquement entre la première d'entre elles et la seconde, mais aussi entre celle-ci et celle-là. Cependant, les noms prédiqués des individus (états de choses, phénomènes, ctc.) impliqués dans une relation symétrique ne renvoient pas uniquement à des actions mutuelles. Ces actions sont égalcment simultanćes. Ainsi, au moment où le nom renvoie à une notion nondurative (intersection, interconnexion, interversion, etc.), les activités de chacun des individus doivent-elles se produire exactement cn même temps. On voit donc que les syntagmes faisant allusion à une relation symétrique s'impliquent aussi du point

relation. Cette notion doit être définic en tcrmes des ensembles de couples ordonnés d'élćments (cf. Cann, 1993: 50). 
de vue temporel. On peut s'en convaincre grâce à l'introduction dans leur structure d'un circonstanciel de temps. La vérité de L'interconnexion de notre réseau et de celui d'outre-Atlantique eut lieu il y a deux ans entraîne nécessairement la fausseté de L'interconnexion du réseau d'outre-Atlantique et du nôtre eut lieu il y a un an. L'unique inférence valable, consécutive à l'énonciation de la première de ces phrases, est L'interconnexion du réseau d'outre-Atlantique et du nôtre eut lieu il $y$ a deux ans.

Cependant, il n'est pas aisé de reconstituer formellement les rapports chronologiques institués par les syntagmes contenant les prédicats nominaux avec le formant inter- et leurs arguments. Ces difficultés sont liées surtout à la diversification aspectuelle des bases dérivationnelles combinables avec le préfixe en question. On peut donc s'attendre légitimement à ce que les relations qui mettent en correspondance les deux arguments établissent entre leurs référents des rapports chronologiques différents. Cette diversité est liée au nombre de valeurs aspectuelles caractérisant les notions abstraites. Il sera admis, par la suite, que ces valeurs sont au nombre de deux. A côté des notions momentanées, il y a des prédicats qui renvoient à des actions et à des états de choses qui s'étendent dans le temps. En effet, à l'opposé des notions non continues (qui n'ont pas de parties et sont indivisibles, cf. intersection, interconnexion ${ }^{3}$, interversion), inter- est librement rattachable aux expressions qui renvoient à des notions duratives. Le sens de celles-ci doit être décrit en termes de l'extension dans le temps (cf. interaction, interdépendance, interchangeabilité, etc.). Du point de vue ontologique, la caractéristique principale de ces unités consiste à renseigner sur les états, donc sur des portions continues délimitées sur l'axe du temps. C'est ainsi qu'une partie temporelle de colère ou de dépendance est toujours de la colère ou de la dépendance (Mourelatos, 1981 : 194196). Il convient donc de mettre en place deux représentations chronologiques distinctes capables de représenter les deux types de noms.

La reconstruction des relations temporelles imposées aux syntagmes par les expressions qui renvoient à des notions non continues est, dans l'optique méthodologique adaptée pour les besoins du présent article, plus simple. Afin de les décrire, il est nécessaire d'introduire deux termes : celui de précédence et celui de coïncidence. Les arguments du premier d'entre eux peuvent être appelés « instants » $(\mathfrak{t})$. lls font partie (symboliquement « $\epsilon »$ ) d'un ensemble plus vaste, qui va être nommé

${ }^{3}$ Puisque les propriétés aspectuelles du sćmantème de interconnexion peuvent soulever ccrtaines controverses, il convient de présenter quelques arguments qui plaident en faveur de son caractère non continu. La base derivationnelle de ce nom - le verbe interconnecter - est définie comme la mise en relation de deux ou plusicurs centres de production ou de consommation d'électricité. Tout portc à croire que c'est unc notion inchoative. On peut donc légitimement s'attendre à ce que interconnexion représente une configuration de plusieurs propriétés aspectuelles. L'information sur le commencement («la mise en relation ») semble être l'élément dominant de cette configuration, ce qui veut dire que d'autres caractéristiques temporelles (p. ex. la période pendant laquuelle les deux objets sont en relation) sont localisées dans l'étendue de la notion de " commencenuent " (cf. Karolak, 1998: 85-86). 
« $T$ » (temps) et ont ceci de particulier que leurs commencements coïncident avec leurs fins. En ce qui concerne les propriétés logiques de la relation de précédence (représentable à l'aide du symbole « <»), il est possbile de les définir de la manière suivante (Nef, 1980:31-34). Pour tous les $\mathrm{t} \in \mathrm{T}$.

2. (i) $\sim\left(t_{i}<t_{i}\right)-$ irréflexive

(ii) $\left(\left(\mathrm{t}_{\mathrm{i}}<\mathrm{t}_{\mathrm{j}}\right) \wedge\left(\mathrm{t}_{\mathrm{j}}<\mathrm{t}_{\mathrm{k}}\right)\right) \rightarrow\left(\mathrm{t}_{\mathrm{i}}<\mathrm{t}_{\mathrm{k}}\right)-$ transitive

(iii) $\left(\mathrm{t}_{\mathrm{i}}<\mathrm{t}_{\mathrm{j}}\right) \rightarrow \sim\left(\mathrm{t}_{\mathrm{j}}<\mathrm{t}_{\mathrm{i}}\right)-$ asymétrique

Il convient de s'interroger sur la disposition des éléments sur cet axe. Celui-ci est construit de telle manière qu'on peut insérer récursivement des points nouveaux entre les éléments successifs de T. Cette propriété s'appelle densité. Comme il a déjà été dit, les événements signifiés par les noms non continus (interconnexion, intersection, interversion, etc.) ne durent pas. Puisqu'ils sont poncutels, il est possible de les apparier avec des instants uniques ( $t$ ) de l'ensemble $\mathrm{T}$. Cet appariement, qui est une fonction (cf. Chierchia et McConnel-Ginet, $2000: 536-539$ ), a un caractère biunivoque. Ceci veut dire qu'il existe entre les événements en question et les instants $t \in T$ une correspondance telle que tout élément du premier ensemble a un correspondant et un seul dans l'autre ${ }^{4}$.

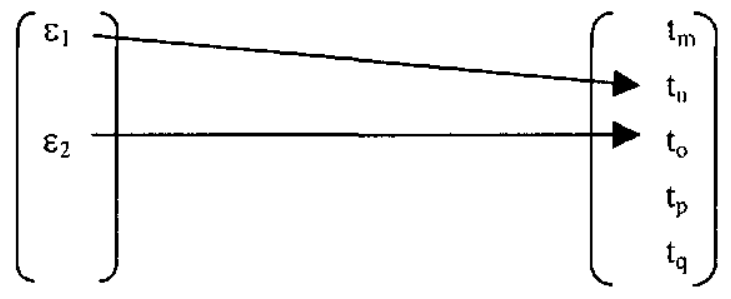

${ }^{4} \mathrm{Cc}$ raisonnement s'appuie sur le présupposé que les noms à préfixe inter-signifient deux actions (situations, événements, états de choses, etc.). En effet. dans les expressions comme La frontière entre les profondeurs abyssales et l univers connu, Le chirurgien a pratiqué la conglutination de deux lèvres d'une blessure ou Janine s'est mariée avec Pierre. les prédicats symétriques précisent la situation de chacun des individus (ou, si l'argument est une notion abstraite, de chaque état de choses, de chaque activité, etc.) impliqués dans la relation (cf. d'un côté les profondeurs abyssales ont une frontière avec quelque chose d'autre el, de l'autre, l'univers conmu a une frontière avec quelgue chosc d'autre, etc.). Une scule notion est donc rapportée aux référents de deux arguments: une première fois, elle est considérée du point de vue de l'un d'entre eux (dans l'exemple ci-dessus, ce qui se passe avec les profondeurs), une seconde fois - du point de vuc de l'autre (ce qui se passe avec l'univers connu). Cette distinction est parallèle au partage de la phrase en thème et propos. Bien que la valeur logique des expressions Janine s'est mariée avec Pierre et Pierre s'est marié avec Janine soit identique, la prédication est faite tantôt sur Janine (ce qui lui est arrivé), à l'exclusion de son partenaire. tantôt au sujet de celui-ci. La langue neutralise parfois cette différence et topicalise les noms de chacun des actants (Pierre et Janine se sont mariés = Ils se sont mariés). 
Du point de vue pragmatique, les syntagmes comme $L$ 'intersection de la route de Nantes et de la route de Niort renseignent donc sur la situation de deux objets individuels. Ce qui est prédiqué du premier d'entre eux (en l'occurrence - à propos de la route de Nantes) est un événement (symbolisé à l'aide de $\varepsilon_{1}$ ) associable à un instant $\left(\mathrm{t}_{\mathrm{e}}\right.$ ') de $\mathrm{T}$ et à lui seul. Il en est de même de l'autre individu auquel l'allusion est faite dans le syntagme ci-dessus (la route de Niort). Sa situation est un événement $\left(\varepsilon_{2}\right)$ rattachable à un autre point ( $t_{\mathrm{e}}$ "') situé sur l'axe du temps $\mathrm{T}$. Maintenant, c'est au prédicat de coïncidence (symboliquement $\cdots \kappa$ ) de prendre la relève. Celui-ci est une relation d'équivalence (ce qui veut dire que la coïncidence est une relation réflexive, symétrique et transitive) dont le domaine de variabilité est formé par tous les instants appartenant à l'ensemble T. Puisque les conditions de vérité de la phrase La route de Nantes et la route de Niort forment une intersection à deux kilomètres d'ici, tandis que la route de Niort et la route de Nantes forment une intersection à dix kilomètres d'ici (moyennant la coréférence de deux ici) ne sont pas possibles à satisfaire, on voit que les deux événements doivent coïncider (être simultanés). Or, là où un syntagme nominal contient un nom ponctuel à préfixe inter-, il y a coïncidence de deux événements signifiés par la base dérivationnelle. Ceci nous conduit à reconnaitre comme valables deux formules: l'une concemant le statut logique du prédicat de coïncidence et l'autre représentant les rapports chronologiques entre les événements signifiés par des prédicats nominaux ponctuels incorporant le formant inter-.

3a. $\kappa=\operatorname{aeq}(\mathrm{T})$

3b. $\left[(\varepsilon 1)=t_{e^{\prime}} \wedge(\varepsilon 2)=t_{e^{\prime}}\right] \rightarrow t_{e^{\prime}} \kappa t_{e^{\prime}}$,

Le statut logique des noms symétriques continus est différent. La caractéristique définitoire de ceux-ci consiste à signifier les états qui durent librement. On voit donc que le recours à des instants uniques de l'ensemble T est incapable de représenter d'une façon adéquate les propriétés temporelles des notions duratives. Ceci ne veut pourtant pas dire que les termes «instant » et « précédence » doivent être d'ores et déjà abandonnés. Bien au contraire, ils seront constamment exploités par la suite. Afin de préciser la nature des rapports chronologiques entre les états de choses dénotés par cette classe de noms prédicatifs incorporant le formant inter-, il est indispensable d'introduire deux nouvelles notions - celle d'intervalle et celle de chevauchement (angl. overlapping). La première d'entre elles est définie en termes des instants. Les intervalles (symbolisés à l'aide de « $\mathrm{i} »)$ ) renvoient à une séquence continue d'instants ordonnés par la relation de précédence (cf. Cresswell, 1977 : 9-10). L'ensemble de tous les intervalles (noté $《 I »)$ est défini dans 4 . Cette formule est révélatrice du caractère continu des intervalles qui ne peuvent pas regrouper des instants non contigus. C'est pour cette raison qu'ils se prêtent mieux à représenter les caractéristiques temporelles des noms duratifs que ne le font les instants uniques. 
4. $I=d_{d f}\left\{i \subseteq T:\left(\left(t_{i} \in i\right) \wedge\left(t_{k} \in i\right) \wedge\left(t_{j}<t_{j}<t_{k}\right)\right) \rightarrow\left(t_{j} \in i\right)\right\}$

Le caractère duratif des intervalles trouve un reflet formel dans la façon dont on a l'habitude de les inscrire : chaque instant enfermé dans un intervalle doit être spécifié, p. ex. $i_{11}=\left[t_{5}, t_{6}, t_{7}, t_{8}, t_{9}, t_{10}, t_{11}\right]$. Par la suite, pour que l'article soit plus concis, seuls les instants initial et ultime seront pris en compte, $p$. ex. in $=\left[t_{5}, t_{11}\right]$. L'appariement des intervalles et de ce qui est prédiqué de chacun des participants à une relation symétrique continue est réalisé d'après les mêmes principes que précédemment. De même que c'était le cas des prédicats nominaux momentanés, la correspondance entre les états de choses et les intervalles est biunivoque. Ce raisonnement part, une nouvelle fois, de l'hypothèse que les noms symétriques font allusion à deux situations, chacune rapportée à un individu (à un état de choses, etc.). En témoigne le syntagme L'interdépendance entre la psychologie et l'éducation qui signifie que la psychologie dépend de quelque chose d'autre et qu'il en est de même de l'éducation. Chacune de ces dépendances doit donc correspondre à un des intervalles $\in \mathrm{I}$ et à lui seul.

Les intervalles sont susceptibles de maintenir différents rapports les uns avec les autres. Ils peuvent, notamment, se précéder, être enfermés (inclus) les uns dans les autres et, enfin, ils peuvent se chevaucher. C'est cette dernière relation (notée habituellement à l'aide du signe ${ }^{\circ}$ ) qui s'avère la plus utile du point de vue de la description des problèmes soulevés ici. En ce qui concerne le statut logique du chevauchement, il représente une relation réflexive, symétrique et non-transitive. Deux intervalles se chevauchent au moment où ils enferment au moins un instant en commun. Cette propriété est symboliquement inscrite dans la formule 5 ci-dessous où « $i_{k} »$ et « $i_{n} »$ représentent deux intevalles distincts.

$$
\text { 5. } i_{k} \circ i_{n} \equiv i_{k} \cap i_{n} \neq \varnothing
$$

Par contre, la succession d'un intervalle à un autre (définie par la formule 6 cidessous $^{5}$ ) ne peut pas être exploitée dans les présentes réflexions. La raison en est que la précédence (dont la succession est la converse) est, cette fois-ci, stricte. Ceci veut dire que les deux intervalles ne peuvent partager aucun élément. Leur partie commune est donc un ensemble vide (symboliquement $i_{k} \cap i_{1}=\varnothing$ ). Les états de

${ }^{5}$ Il existe une profonde différence entre la notion de précédence exploitée lors de la description des instants et de l'ensemble qu'ils forment et celle à laquelle on fail appel pendant l'analyse des intervalles. Alors que la première est connectable (ce qui permet à deux instants de coïncider, c.à.d. d'avoir lieu exactement en même temps, cf. Schwarzschild. Wilkinson, $2002: 37-38$ ), cette propriété logique fait défaut à la seconde. Cette différence découle de la nécessité de fairc une démarcation rigoureuse, lorsqu'on parle d'intervalles, entre le chevauchement de ceux-ci (dont la condition est que les deux ensembles aicnt au moins un instant en commun) et la situation où les deux périodes de temps forment des ensembles disjoints (c.à.d. où ils n'ont aucune partie en commun). Le non-respect de cette contrainte aurait fini par mettre sur un pied d'égalité les deux relations. Une d'entre elles serait donc inutile. 
choses mis en rapport par la relation de précédence stricte ne peuvent donc pas être simultanés. Dès lors qu'on se rend compte qu'une séparation complète des intervalles ne peut pas être pratiquée là où l'on a affaire à la symétrie, on comprend pourquoi la précédence stricte est inapplicable ici.

$$
\text { 6. } \mathrm{i}_{\mathrm{k}}<\mathrm{i}_{\mathrm{n}} \equiv\left(\forall \mathrm{t} \in \mathrm{i}_{\mathrm{k}} \wedge \forall \mathrm{t}^{\prime} \in \mathrm{i}_{\mathrm{n}}\right) \rightarrow \mathrm{t}<\mathrm{t}^{\prime}
$$

Puisque les états (de même que tout ce qui est dénoté par des expressions prédicatives continues) ne peuvent pas être ponctuels, mais qu'ils doivent s'étendre, il est incorrect de leur faire correspondre des instants uniques. Par contre, ils peuvent facilement revêtir la forme d'intervalles appartenant à l'ensemble I (cf. Zucchi, 1999 : 180-184 et 202-208). C'est de cette manière qu'il faut décrire les prédispositions dont il est question dans L'interchangeabilité des syntagmes nominaux et des pronoms démonstratifs. Dans une optique pragmatique, cette construction affirme deux choses - l'aptitude des pronoms à remplacer les syntagmes et l'aptitude des syntagmes à se substituer aux pronoms. Par la suite, la prédisposition (qui est une notion durative) rapportée à des syntagmes nominaux va correspondre à l'intervalle $i_{\mathrm{s}}$. L'étape suivante consiste en une spécification des instants enfermés dans $i_{\mathrm{s}}$. Puisque la capacité à remplacer quelque chose n'est pas momentanée, le nombre de ces instants doit être supérieur à un. Soit donc $i_{s}=\left[t_{k}, t_{q}\right]$. Le mécanisme de la délimitation des instants et la forme qu'épouse la représentation temporelle de ce qui est prédiqué des pronoms démonstratifs dans l'exemple ci-dessus sont analogues. L'intervalle qui encadre leur aptitude à être substitués à des syntagmes sera, conventionnellement, associée à un autre ensemble continu $\mathrm{i}_{\mathrm{p}}$. Celui-ci s'étend à la période enfermée entre les instants $\left[t_{j}, t_{p}\right]$.

La nature des formations à préfixe inter- (de même que celle de tous les prédicats symétriques) exige la simultanéité des actions (des états de choses, des événements, etc.) des individus qui participent à la relation signifiée par la base dérivationnelle. Au moment où cette relation est continue, les activités de chaque participant doivent avoir une partie commune. Or, il résulte de l'interchangeabilité des syntagmes nominaux et des pronoms démonstratifs que les états signifiés par l'interchangeabilité ne peuvent pas être complètement disjoints. A un certain moment (ou pendant une certaine période), ils doivent avoir lieu, l'un en même temps que l'autre. C'est conforme à la définition 5 ci-dessus. A l'opposé de ce qui se passe là où la relation n'est pas continue, les deux états ne doivent pas obligatoirement coïncider, c.à.d. être associés à un instant unique (Giermak-Zielińska, $1997: 272$ ). C'est donc la notion de chevauchement qui s'avère optimale pour les besoins de la représentation des rapports temporels institués par les notions continues dont les équivalents linguistiques se font précéder de inter-. Du point de vue chronologique, les syntagmes dont le noyau est un nom duratif incorporant ce formant ont la propriété de renvoyer à l'intervalle pendant lequel les états signifiés par la base dérivationnelle persistent, l'un aussi bien que l'autre. Les formules 7a et 
$7 \mathrm{~b}$ rendent compte du cadre temporel caractéristique de l'interchangeabilité des syntagmes nominaux et des pronoms démonstratifs.

7a. $i_{p} \cap i_{5} \neq \varnothing$

7b. $i_{p}{ }^{\circ} i_{s}=\left[t_{k}, t_{p}\right]$

A côté du chevauchement, c'est la relation d'inclusion qui est susceptible d'être exploitée à bon escient dans l'analyse des rapports entre les états signifiés par les prédicats duratifs incorporant le préfixe inter-. En effet, une des dépendances (p. ex. celle de la psychologie) dont il est question dans l'interdépendance de la psychologie et de l'éducation peut avoir commencé avant et avoir fini après la période de dépendence de l'éducation. Ce qui caractérise la psychologie peut être donc situé à l'intérieur de l'intervalle $i_{p}=\left[t_{k}, t_{w}\right]$. De son côté, la durée de ce qui est prédiqué de l'éducation, associable à la période $i_{\mathfrak{e}}=\left[t_{p}, t_{t}\right]$, peut être entièrement enchâssée dans l'intervalle caractérisant le cadre temporel du premier état. Ainsi, les deux états sont, pendant un certain temps, coextensifs. En termes de la théorie des ensembles, ceci signifie qu'ils ont plusieurs éléments en commun. C'est, une nouvelle fois, conforme à la définition 5 . Il en est ainsi, car l'unique condition imposée aux rapports temporcls caractéristiques des états dénotés par les noms continus incorporant le préfixe inter- est l'existence d'une partie commune. L'inclusion d'un intervalle dans la durée d'un autre est, dans cette perspective, un cas de figure de la relation de chevauchement. On obtient donc deux équations.
8a. $\left[\mathrm{t}_{\mathrm{k}}, \mathrm{t}_{\mathrm{w}}\right] \cap\left[\mathrm{t}_{\mathrm{p}}, \mathrm{t}_{\mathrm{t}}\right]=\left[\mathrm{t}_{\mathrm{p}}, \mathrm{t}_{\mathrm{t}}\right]$
8b. $\left[\mathrm{t}_{\mathrm{p}}, \mathrm{t}_{\mathrm{t}}\right] \subseteq\left[\mathrm{t}_{\mathrm{k}}, \mathrm{t}_{\mathrm{w}}\right]$.

L.c raisonnement qui précède, en dépit de son caractère abstrait, a des conséquences tout à fait utilitaires. Elles concernent la façon dont on a l'habitude de décrire les problèmes relatifs à l'affixation dans les manuels d'enseignement universitaire. Le recours exclusif à l'énumération des formants et de lcurs paraphrases ne suffit pas à présenter les conséquences linguistiques de l'agglutination de certains formants à leurs bases dérivationelles. Ainsi les apprenants sont-ils incapables de connaître l'emploi réel des éléments en question. C'est une imperfection didactique. Il n'est donc pas étomnant de postuler un autre mode de la présentation des affixes. En ce qui concerne inter-, ce traitement doit être conçu de sorte à :

- montrer quelle est l'influence de cet affixe sur le sens du nom. Il est question ici du passage d'une relation non symétrique (l'action de $x$ sur $y$ ) à une situation où les deux participants sont engagés à un même degré dans la réalisation du procès signifié par la base dérivationnelle (l'interaction de $x$ et de $y$ ). yl est donc désirable d'expliquer, ne serait-ce que d'une façon approximative, quelle est la nature de la symétrie ; 
- montrer l'impact des noms ainsi modifiés sur les caractéristiques structurales des syntagmes. Il s'agit de la nécessité de comprimer les arguments à un pronom pluriel ou bien de coordonner les noms des entités participant à la relation (l'interdépendance de la psychologie et de l'éducation - leur interdépendance). Il est également souhaitable de signaler la possibilité de la permutation des arguments ;

-- montrer quels sont les rapports temporels entre les actions et les états de choses signifiés par les noms avec le préfixe inter-. Ces relations sont liées, il faut le souligner, à la diversité des caractéristiques aspectuelles de la base dérivationnelle modifiée par le formant en question.

\section{BIBLIOGRAPHIE}

Bańczerowski J. (1980), Systems of Semantics and Syntax. A determinational theory of language, PWN, Warszawa-Poznan.

Cann R. (1993), Formal Semantics. An Introduction, Cambridge University Press, Cambridge.

Chierchia G., McConnel-Ginet S. (2000), Meaning and Grammar : an Introduction to Semantics, The MIT Press, Cambridge, Massachusetts-London.

Cresswell M. (1977), Interval semantics and logical words. (in :) C. Rohrer, ed., On the Logical Analysis of Tense and Aspect, Tübinger Beiträge zur Linguistik 80, Tübingen, pp. 7-29.

Giermak-Ziclinska T. (1997), L'expression de la continuation en français et en polonais (in :) K. Bogacki. T. Giermak-Zielińska, eds., Espace et temps dans les langues romanes et slaves. Actes du VIII colloque de linguistique romane et slave. Varsovie. 19-21 septembre 1996, L'Institut de Philologie Romane - Université de Varsovic, Warszawa, pp. 267-276.

Jackendoff R. (1975), Morphological and Semantic Regularities in the Lexicon, Language 51 (3), pp. 639-671.

Jalenques P. (2002), Étude sémantique du préfixe RE en français contemporain : à propos de plusieurs débats en morphologie dérivationnelle, Languc française 133, pp. 74-90.

Karolak S. (1998), Catégorisation sémantique des verbes et aspect. Lingua Posnaniensis XL, pp. $71-87$.

Marek W.. Onyszkiewicz J. (2001), Elementy logiki i teorii mmogości wadaniach, Wydawnictwo Naukowe PWN, Warszawa.

Mourelatos A.P. (1981), Events, Processes and States, (in :) P. J. Tedeschi et A. Zuenen, eds., Syntax and Semantics, vol. 14 : Tense and Aspect, New York Academic Press, New York, pp. 191-212.

$\mathrm{Nef} F .(1980)$, Les verbes aspectucls du français : remarques sémantiques et esquisse d'un traitement formel, Semantikos 4 (1), pp. 11-46

Schwarzschild R., Wilkinson K. (2002), Quantifiers in Comparatives : A Semantics of Degree Based on Intervals, Natural Language Semantics 10 (1), pp. 1-41.

Zucchi S. (1999), Incomplete Events. Intensionality and Imperfective Aspect, Natural Language Semantics 7 (2), pp. 179-215. 